\title{
New molecular settings to support in vivo anti-malarial assays
}

Noemí Bahamontes-Rosa*, Ane Rodriguez Alejandre, Vanesa Gomez, Sara Viera, María G. Gomez-Lorenzo, Laura María Sanz-Alonso and Alfonso Mendoza-Losana

\begin{abstract}
Background: Quantitative real-time PCR ( $(\mathrm{PCCR})$ is now commonly used as a method to confirm diagnosis of malaria and to differentiate recrudescence from re-infection, especially in clinical trials and in reference laboratories where precise quantification is critical. Although anti-malarial drug discovery is based on in vivo murine efficacy models, use of molecular analysis has been limited. The aim of this study was to develop qPCR as a valid methodology to support pre-clinical anti-malarial models by using filter papers to maintain material for $\mathrm{QPCR}$ and to compare this with traditional methods.
\end{abstract}

Methods: FTA technology (Whatman) is a rapid and safe method for extracting nucleic acids from blood. Peripheral blood samples from mice infected with Plasmodium berghei, P. yoelii, or P. falciparum were kept as frozen samples or as spots on FTA cards. The extracted genetic material from both types of samples was assessed for quantification by qPCR using sets of specific primers specifically designed for Plasmodium 18S rRNA, LDH, and CytB genes.

Results: The optimal conditions for nucleic acid extraction from FTA cards and qPCR amplification were set up, and were confirmed to be suitable for parasite quantification using DNA as template after storage at room temperature for as long as 26 months in the case of P. berghei samples and 52 months for P. falciparum and P. yoelii. The quality of DNA extracted from the FTA cards for gene sequencing and microsatellite amplification was also assessed.

Conclusions: This is the first study to report the suitability of FTA cards and QPCR assay to quantify parasite load in samples from in vivo efficacy models to support the drug discovery process.

Keywords: Malaria, Plasmodium falciparum, Plasmodium yoelii, Plasmodium berghei, Quantitative real-time PCR, QPCR, FTA

\section{Background}

In anti-malarial clinical trials discerning between mixed infections, resistance, re-infection and recrudescence, is crucial to establish the efficacy of new anti-malarials. The World Health Organization highly recommends the use of molecular analysis techniques, like PCR, to assist in distinguishing recrudescence from re-infection [1], and several methods have been implemented so far for this purpose [2]. In clinical trials, a cheap and long-term storage method is used for biological samples. The FTA technology (Whatman) consists of index-sized cards

*Correspondence: noemi.r.bahamontes-rosa@gsk.com Diseases of the Developing World, GlaxoSmithKline, Tres Cantos, 28760 Madrid, Spain developed for collection and storage of organic samples from field isolates. FTA cards are routinely used to support clinical trials because they are safe and easy to transport at room temperature between laboratories, and the genetic material of the parasite remains unchanged in the spot sample for long-term storage [3, 4]. However, no research has been performed so far to translate the use of this technique to mouse malaria models. In drug discovery, the most common murine Plasmodium species used are Plasmodium berghei and P. yoelii and, a closer subrogate for human parasite infection, the humanized mouse model with P. falciparum [5-7].

Among molecular detection techniques, quantitative real-time PCR (qPCR) has demonstrated a high sensitivity for detection of low parasite burdens, and is now

\section{Biomed Central}

(C) 2016 Bahamontes-Rosa et al. This article is distributed under the terms of the Creative Commons Attribution 4.0 International License (http://creativecommons.org/licenses/by/4.0/), which permits unrestricted use, distribution, and reproduction in any medium, provided you give appropriate credit to the original author(s) and the source, provide a link to the Creative Commons license, and indicate if changes were made. The Creative Commons Public Domain Dedication waiver (http://creativecommons. org/publicdomain/zero/1.0/) applies to the data made available in this article, unless otherwise stated. 
commonly used as a method to confirm diagnosis of malaria, especially in clinical trials and in reference laboratories where precise quantification is critical [8-10]. This is the first study to report parasite identification and quantification using FTA blood spots to support pre-clinical animal models. It was designed to identify the adequate molecular settings that may be encountered across laboratories to support anti-malarial in vivo assays.

\section{Methods}

\section{Parasites and animal models}

The Plasmodium species tested as tool organisms for drug screening were the human pathogen $P$. falciparum and the mouse malaria parasite species $P$. yoelii and $P$. berghei.

Plasmodium falciparum Pf3D7 $7^{0087 / \mathrm{N} 9}$ was maintained continuously in vivo in non-myelodepleted NOD-scid IL2R gamma-null mice obtained from Charles River Laboratories, engrafted with human erythrocytes [5]. Plasmodium yoelii and P. berghei were maintained by in vivo passage on CD1 mice purchased from Harlan Laboratories. Parasitaemia from mouse malaria models was routinely monitored on peripheral blood by flow cytometry [11]. Protocols were approved by the DDW Ethical Committee on Animal Research, performed at the DDW Laboratory Animal Science facilities accredited by AAALAC, and conducted according to European Union regulations and GlaxoSmithKline policy on the care and use of animals.

\section{Biological material}

Plasmodium falciparum-infected blood samples were taken from the tail vein of the NOD-scid IL2R gammanull engrafted mice, and blood samples infected with $P$. berghei or P. yoelii were taken from CD1 mice. Parasitaemia was measured by flow cytometry. To establish the limit of detection (LOD), P. falciparum blood samples were serially diluted tenfold using fresh $\mathrm{AB}+$ human erythrocyte concentrates generously donated by the Spanish Red Cross blood bank, and P. berghei and P. yoelii blood samples were diluted with uninfected blood from a donor CD1 mouse. Non-infected human RBC samples were included as negative controls for $P$. falciparum samples, and mouse blood samples as negative controls for $P$. berghei and $P$. yoelii. Fifty $\mu \mathrm{l}$ from all samples were immediately stored at $-80{ }^{\circ} \mathrm{C}$, and $20 \mu$ l spotted onto an FTA card (GE Healthcare), dried for $3 \mathrm{~h}$ at room temperature (RT), and stored at RT from 1 day up to 26 months for $P$. berghei samples and for 52 months for P. yoelii and P. falciparum for further DNA extraction. The lowest concentration of DNA that tested positive in all replicates was considered the LOD.

\section{DNA extraction}

To extract DNA, the DNeasy Blood and Tissue Kit (Qiagen) was used. For frozen samples, the manufacturer recommendations were followed. For DNA isolation from the FTA cards, the complete disk was punched out from each filter paper and incubated with the lysis buffer provided by the manufacturer at $56{ }^{\circ} \mathrm{C}$ for $1 \mathrm{~h}$, followed by incubation for $10 \mathrm{~min}$ at $70{ }^{\circ} \mathrm{C}$ in shaking conditions. Lysed infected red blood cells (iRBC) were then placed onto the purification column provided, and purification continued according to the standard protocol suggested by the manufacturer.

\section{Genes used}

The Plasmodium 18S ribosomal RNA (18S rRNA), lactate dehydrogenase (LDH), and cytochrome B (CytB) genes chosen for the study encode highly conserved proteins and are considered as housekeeping genes in detection and quantification studies [12-14]. Gene sequences were retrieved from PlasmoDB and used as templates to design qPCR primers using Primer Express Software v.1.5 (Applied Biosystems). Primer sequences are described in Table 1 . Primers already reported in the literature for $P$. falciparum LDH, P. yoelii 18S rRNA, and P. berghei $\mathrm{LDH}$ and $\mathrm{CytB}$ primers were also used in this study [12-15].

\section{Quantitative real-time PCR}

Quantitative real-time PCR was performed using an ABI PRISM 7500 fast sequence detection system (Applied

\section{Table 1 Genes and primer sequence}

\begin{tabular}{|c|c|}
\hline \multicolumn{2}{|c|}{ P. falciparum } \\
\hline \multirow[t]{2}{*}{ 18S rRNA } & Pf_18S rRNA-F: AATAACAATGCAAGGCCAATTT \\
\hline & Pf_18S rRNA-R: CTGCAACAATTTTAATATACGC \\
\hline \multirow[t]{2}{*}{ LDH } & Pf_LDH-F: AAACCCAGTAGATGTTATGGTAC \\
\hline & Pf_LDH-R: CATTTTATTTCCATGAGCACCTAC \\
\hline \multirow[t]{2}{*}{ CytB } & Pf_CytB-F: ACTTGTTATCCTCTATTCCAGTAGCAGTAA \\
\hline & Pf_CytB-R: AATCGTTTTATTGTAGGATCACTCACA \\
\hline \multicolumn{2}{|l|}{ P. yoelii } \\
\hline \multirow[t]{2}{*}{185 rRNA } & Py_18S rRNA-F: GCAACTCACTTGGCTAGATTCTT \\
\hline & Py_18S rRNA-R: CGGCTTTAACTGTTTGCTTTG \\
\hline \multirow[t]{2}{*}{ LDH } & Py_LDH-F: GCTCGACTTTATTAGAAGGCCAAT \\
\hline & Py_LDH-R:TCGATTACTTGTTCTACACCATTACCA \\
\hline \multirow[t]{2}{*}{ CytB } & Py_CytB-F:TTTACATTTACATGGTAGCACTAATCCTTT \\
\hline & Py_CytB-R: CCTTTTACATCAAGACTTAATAGATTTGGA \\
\hline \multicolumn{2}{|l|}{ P. berghei } \\
\hline \multirow[t]{2}{*}{ 18S rRNA } & Pb_18S rRNA-F: AAGCATTAAATAAAGCGAATACATCCTTAC \\
\hline & Pb_18S rRNA-R: GGAGATTGGTTTTGACGTTTATGTG \\
\hline \multirow[t]{2}{*}{ LDH [15] } & Pb_LDH-F: GGAAGGAGGTTCACAAGCAGGT \\
\hline & Pb_LDH-R:TATGCTCTCAGCCAAGTCTGCC \\
\hline \multirow[t]{2}{*}{ CytB [14] } & Pb_CytB-F:TGGGGACAAATGAGTTACTGG \\
\hline & Pb_CytB-R: CAGTGTATCCTCCACATAACCAA \\
\hline
\end{tabular}

$L D H$ lactate deshydrogenase, $C y t B$ cytochrome b 
Biosystems). Reagents used included Sybr green JumpStart Taq ReadyMix (Sigma); $250 \mathrm{nM}$ forward primer; $250 \mathrm{nM}$ reverse primer; and distilled sterile water up to a total reaction volume of $13+2 \mu \mathrm{l}$ of the genetic material. Reaction mixtures were prepared at RT in a 96-well optical reaction plate (Applied Biosystems) covered with optical adhesive covers (Applied Biosystems). The thermal cycling conditions were $50{ }^{\circ} \mathrm{C}$ for $2 \mathrm{~min}, 95{ }^{\circ} \mathrm{C}$ for $10 \mathrm{~min}$ and 40 cycles of $95{ }^{\circ} \mathrm{C}$ for $15 \mathrm{~s}$ and $60{ }^{\circ} \mathrm{C}$ for 1 min. Amplification of each gene was done in duplicate, and the average value was taken as the result. Ct values are inversely proportional to the amount of template in the sample and were used to quantify the relative amount of target PCR product present in each reaction. Only duplicate $\mathrm{Ct}$ values with standard deviation within \pm 1.5 $\mathrm{Ct}$ were accepted. All qPCR assays were run including the Non-Template Control (NTC), and melting curve analysis was always performed at the end of each assay as a specificity control.

\section{Parasite genotyping}

Genotyping of $P$. falciparum $\mathrm{Pf} 3 \mathrm{D} 7^{0087 / \mathrm{N} 9}$ strain was done by amplification of the microsatellite regions from DNA extracted from the FTA cards. The technique, based on the multiple and species-specific repetition of microsatellite sequences, was described by $\mathrm{Su}$ et al. in 1998 using specific primers labeled with FAM (FFF: TAACGT TACATTATGTTTTA-FAM and FFR: ATATGGTAT TGCGCTTTTA) [16].

\section{Amplification and sequencing of $P$. falciparum CytB gene}

The protocol used was an adaptation from the one reported by Korsinczky et al. [17]. The CytB gene was amplified from DNA extracted from the FTA cards using primers F CYTB and R CYTB that anneal outside the ORF (primer sequences in Table 2). PCR was performed using $2 \mu \mathrm{l}$ of DNA as template. PCR products were purified and then sequenced using the ABI Prism Big-Dye Terminator kit (Applied Biosystems). Nine internal primers (forward and reverse) were then used to sequence the amplified products (Table 2).

\section{Results}

\section{Suitability of FTA for in vivo anti-malarial studies}

The value of FTA cards for collecting and storing samples from in vivo animal anti-malarial studies was assessed. DNA from the filter papers were recoverd using a silica-membrane-based column method. Optimal DNA yield and quality for direct $\mathrm{qPCR}$ analysis was obtained following the protocol described here. In order to further assess the quality of the nucleic acids extracted from FTA cards, the species-specific repetition of microsatellite was successfully amplified, giving the
Table 2 Primer for amplification and sequencing of the $P$. falciparum CytB gene

\begin{tabular}{ll}
\hline Primer for amplification & \\
FCYTB & CCTGATTATCCAGACGCTTTAAATGG \\
R CYTB & CATGTCTTGCTAACGGCTTGTACGG \\
Primer for sequencing & \\
BC1 & AACGGTGTATTTTTAGCAAGTGG \\
BC2 & GAATAAAAAATATTATTCCTAAAAGG \\
BC3 & CAAATGAGTTATTGGGGTCAAC \\
BC4 & TCATAAATAAAATCAATCCAGATATCC \\
BC5 & TATCCAAATCTATTAAGTCTTGATG \\
BC6 & AATGCTGTATCATACCCTAAAGG \\
BC7 & GGTGCTAGAGATTATTCTGTTCC \\
BC8 & TGTTCTGCTAATAAGAATAATAATTG \\
BC9 & AACACATTATGATTACAGCTCC \\
\hline
\end{tabular}

same pattern as previously reported for the $P$. falciparum strain used in the mouse model (Fig. 1a) [18]. Using the same extracted genetic material, the Pf CytB gene was sequenced, finding no mutations as compared to the CytB 3D7 P. falciparum genome sequence M76611 (PlasmoDB). The peaks from the electropherograms were single and clear for each nucleotide without uncertainty, with a low background line (Fig. 1b). These results showed that the extracted genetic material had enough quality to support identification of specific resistance determinants in long DNA stretches or to assess identification of mixed infections.

\section{qPCR setup conditions}

The optimal qPCR amplification conditions were set up using DNA obtained from tenfold serial fresh blood samples diluted with human erythrocyte concentrates for $P$. falciparum and with uninfected blood from a donor mouse for $P$. berghei and $P$. yoelii. Each assay was performed in duplicate, and efficiency of amplification with the primer sets was calculated. A linear regression plot was generated and the slope, Y-intercept, and $r^{2}$ value were calculated. Data shown in Table 3 confirm that all primers showed suitable conditions for parasite quantification and efficiencies ranging from 94.88 to $50.46 \%$, with $\mathrm{r}^{2}$ ranging from 0.99 to 0.92 .

\section{Parasite LOD calculation by $\mathrm{qPCR}$}

For parasite detection, LDH, 18S rRNA, and CytB genes were evaluated using the corresponding strain-specific primers shown in Table 1. The LOD of the qPCR assay was determined from DNA extracted from frozen blood samples and embedded in FTA cards. All assays were performed in duplicate, and the LOD was established as the highest $\mathrm{Ct}$ value at which the lowest parasite 


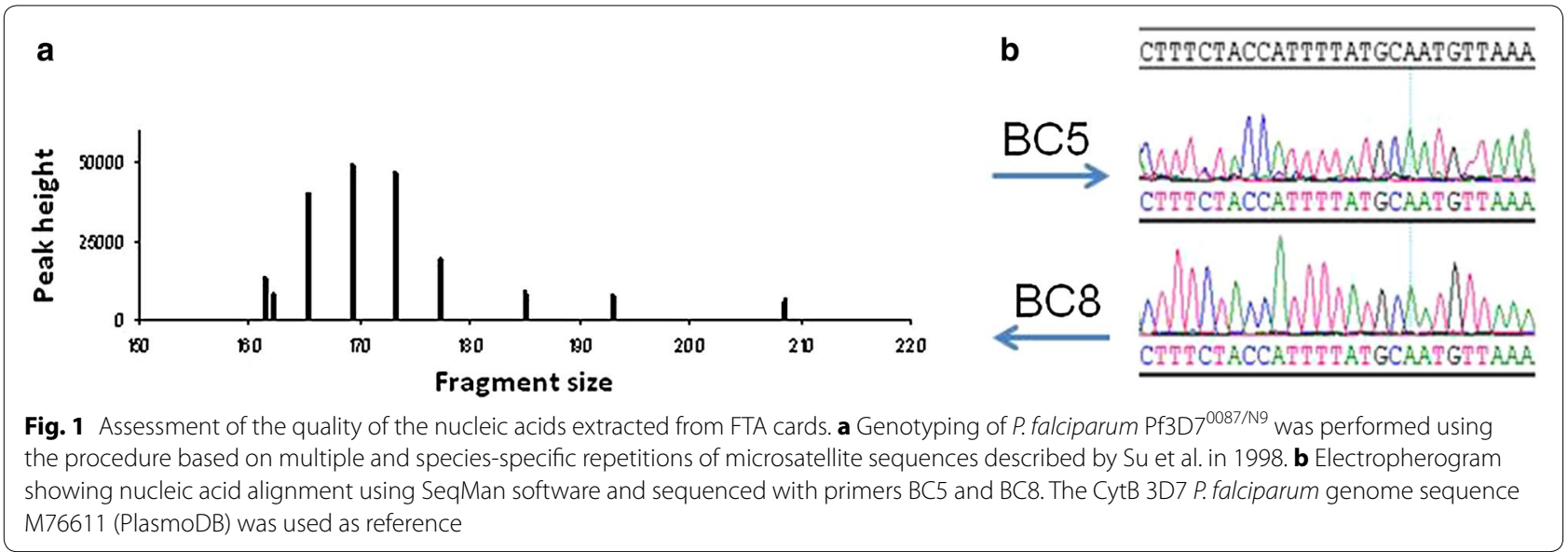

Table 3 qPCR assay performance

\begin{tabular}{lcccc}
\hline Gene & Slope & Y-Inter & $\mathbf{R}^{\mathbf{2}}$ & \% Eff \\
\hline P. falciparum & & & & \\
LDH & -3.77 & 27.03 & 0.99 & 84.33 \\
CytB & -3.80 & 16.49 & 0.99 & 83.38 \\
18S rRNA & -3.56 & 5.69 & 0.99 & 90.92 \\
P. yoelii & & & & \\
LDH & -3.45 & 21.62 & 0.95 & 94.88 \\
CytB & -3.70 & 13.97 & 1.00 & 86.28 \\
18S rRNA & -3.73 & 16.65 & 0.99 & 85.49 \\
P. berghe & & & & \\
LDH & -4.27 & 23.30 & 0.96 & 71.43 \\
CytB & -5.64 & 22.53 & 0.92 & 50.46 \\
18S rRNA & -4.55 & 25.42 & 1.00 & 65.93 \\
\hline
\end{tabular}

The efficiency of each qPCR was calculated with the formula:

Efficiency $=-1+10(-1 /$ slope) (http://www.thermoscientificbio.com/webtools/ qpcrefficiency/), which uses the slope produced by a qPCR standard curve. qPCR efficiency was validated by comparing the slope of the standard curve to the theoretical optimum of -3.32 which reflects $100 \%$

concentration was detected and expressed as $\log 10$ parasites/ $\mu \mathrm{l}$. Only $\mathrm{Ct}$ values below 40 and duplicate $\mathrm{Ct}$ values with standard deviation within $\pm 1.5 \mathrm{Ct}$ were considered. The mean LOD values are given in Table 4 and varied depending on the source and genes used. Assay fluctuation included variation in both nucleic acid extraction and amplification. DNA from FTA cards was successfully obtained and even a parasite burden lower than one parasite/ $\mu \mathrm{l}$ (negative $\log 10$ parasites $/ \mu \mathrm{l}$ ) was detected with $P$. falciparum $\mathrm{Cyt} \mathrm{B}$ gene. Similar detections were obtained in $P$. berghei with the CytB and $18 \mathrm{~S}$ rRNA genes and in $P$. yoelii with the $18 \mathrm{~S}$ rRNA gene. When LOD values were compared between frozen samples and FTA cards, a lower detection limit was seen for the CytB gene in samples stored in filter paper. For frozen samples, higher sensitivity was seen for the LDH and 18S rRNA genes.
Table 4 Limit of detection ( $\log 10$ parasites/ $\mu \mathrm{l})$

\begin{tabular}{|c|c|c|}
\hline Gene & DNA frozen & DNA FTA \\
\hline \multicolumn{3}{|c|}{ P. falciparum } \\
\hline $\mathrm{LDH}$ & 2.2 (1.1 to 3.2 ) & 4.9 (4.5 to 5.2$)$ \\
\hline CytB & 1.9 (1.1 to 2.6$)$ & 0.4 ( -0.8 to 1.5$)$ \\
\hline 18S rRNA & 1.9 (0.1 to 3.6) & 3.9 (2.5 to 5.2 ) \\
\hline \multicolumn{3}{|l|}{ P.yoelii } \\
\hline LDH & 0.8 (0.8 to 0.8 ) & 2.2 (0.2 to 4.2$)$ \\
\hline CytB & 2.8 (0.8 to 4.8 ) & 1.5 (0.9 to 2.2 ) \\
\hline 18S rRNA & $0.3(-0.2$ to 0.8$)$ & 1.2 (0.2 to 2.2 ) \\
\hline \multicolumn{3}{|l|}{ P. berghei } \\
\hline $\mathrm{LDH}$ & 0.9 (0.9 to 0.9 ) & 1.8 (1.3 to 2.3 ) \\
\hline CytB & $0.9(-0.1$ to 1.9$)$ & 0.8 (0.3 to 1.3$)$ \\
\hline $18 \mathrm{~S}$ rRNA & $0.4(-1.1$ to 1.9$)$ & 2.8 (2.3 to 3.3 ) \\
\hline
\end{tabular}

Detection limit of $\log 10$ parasites/ $\mu$ l expressed as the median of the experiments (min-max)

\section{FTA long-term storage}

The suitability of FTA cards for long-term storage of in vivo samples was addressed. FTA cards with Plasmodium samples were stored before DNA extraction at RT and protected from light and humidity for 52 months for $P$. yoelii and P. falciparum samples and for 26 months for $P$. berghei. The LOD $(\log 10$ parasites $/ \mu \mathrm{l})$ achieved in these cards using the $\mathrm{CytB}$ gene was 3.8 for $P$. yoelii, 3.2 for $P$. falciparum, and 1.3 for $P$. berghei. Figure 2 shows the correlation of the $\mathrm{Ct}$ values obtained from DNA extracted from FTA cards stored and from samples processed directly after FTA embedding without storage. $P$. yoelii and $P$. falciparum $\mathrm{Ct}$ values were similar at high parasitaemia levels or Ct under 24 and 26 respectively. For $P$. berghei, a linear correlation existed in all samples tested. The DNA yielded from the FTA cards stored met the quality requirements for qPCR, but rendered a lower LOD than the ones recently embedded. 


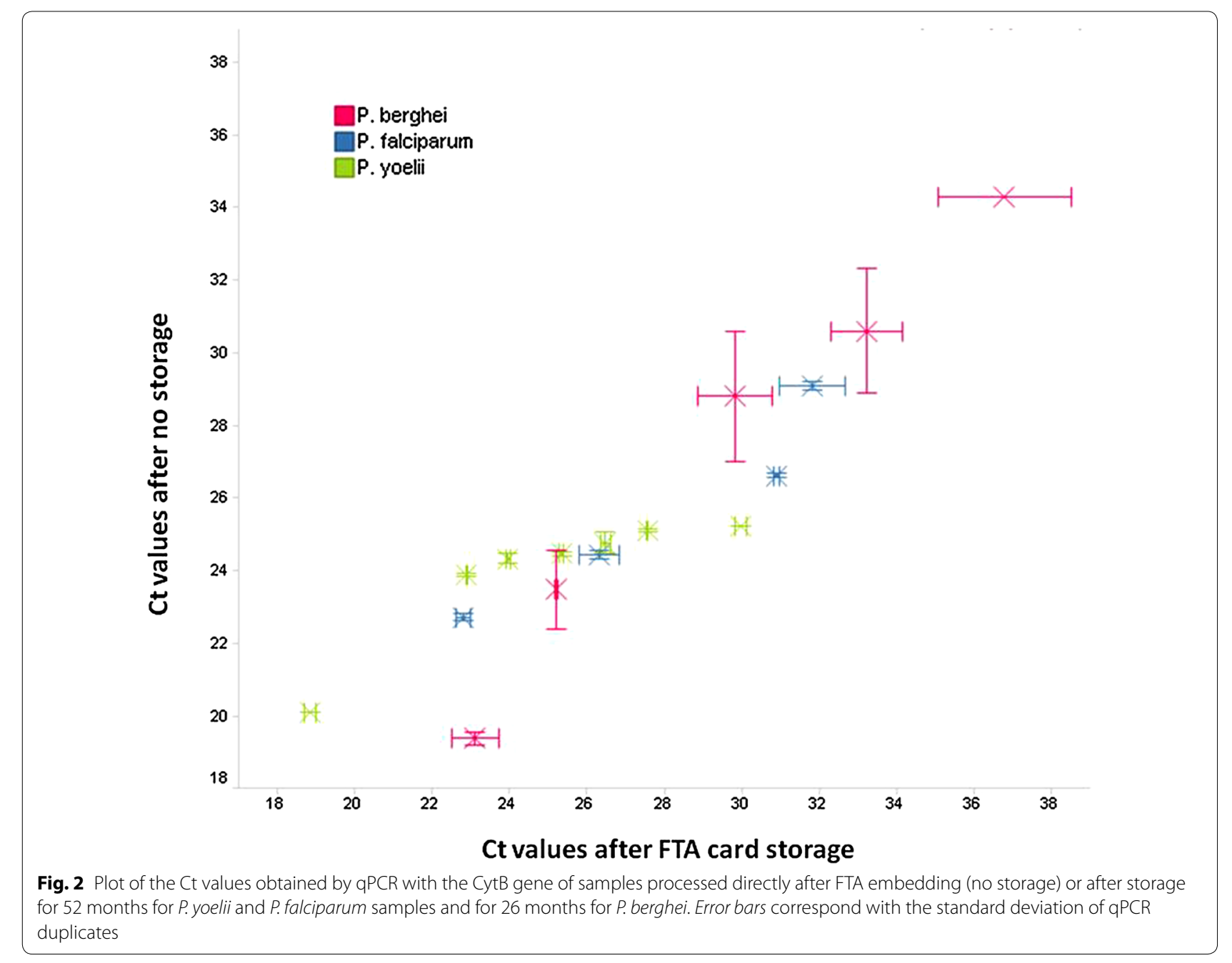

\section{Discussion}

Determination of parasitaemia by flow cytometry is accurate with a LOD of $0.0009 \times 10^{6}$ parasites/ $\mu$ l but has the limitation of sample storage, and although microscopy is still considered the gold standard, it has a reduced throughput. Detection of Plasmodium DNA by amplification techniques based on PCR from dried blood on filter paper has been effectively used for sexual and asexual parasites even in studies conducted over decades $[3,9,19$, 20]. Studies performed with filter paper stored over long time periods and under different conditions have shown variable results as regards sensitivity for parasite detection [21]. Moreover, previous cross-platform work and crossstudy comparisons already showed discrepancies on LOD in P. falciparum studies [22]. The study discussed here is the first to report use of filter paper with samples from mouse malaria models to support the early drug discovery process. The advantage of using FTA cards for in vivo assays has been shown, as they allow for sample storage at
RT even for long time. In the present study, after extended storage periods at RT, although the LOD range was lower than for newly generated cards, the DNA extracted was sufficient for qualitative parasite detection, thus providing an advantage for retrospective studies.

The preanalytical steps of $\mathrm{qPCR}$, and mainly DNA extraction, are extremely important because the quality of the genetic material collected impacts all downstream activities. Previous studies investigated different methods to optimize DNA extraction from filter papers [23-25]. In this study, and optimal DNA quality was obtained using the in-column silica membrane-based method. The qPCR technique has some limitations as routine methodology, including long sample processing, but was more sensitive than microscopy [10], powerful for detecting genes related to drug resistance, and amenable to parasite genotyping [2]. All these properties are critical in preclinical settings where access to and analysis of the original samples is crucial to identify potential drug failures. 
Malaria-specific qPCR is being increasingly used because of its high sensitivity, speciation, and quantification of malaria parasites. In this study, although some qPCR efficiencies were slightly outside the desirable efficiency range of 90-105\%, quantification of Plasmodium parasites through qPCR with complete specificity has been shown even for samples with very low parasite densities using a set of primers for the most common analysed genes from murine Plasmodium species (P. berghei and $P$. yoelii) and the human pathogen studied in the humanized mouse model (P. falciparum).

\section{Conclusions}

This study has reported for the first time complete primer sets for parasite detection and quantification to be used in in vivo anti-malarial trials, and demonstrated the feasibility of using filter paper to collect and store in vivo animal samples.

The results of this study suggest that FTA cards are a practical and cost-effective, reliable source of Plasmodium DNA amenable to parasite detection and practical for studies of anti-malarial resistance genes and species identification. FTA cards require a low volume of sample and are easy to store over time. This technique represents a practicable tool for preclinical trials with anti-malarial drugs.

\section{Authors' contributions}

NB conceived and designed the study, carried out laboratory experiments, conducted data management and data analysis, and wrote the manuscript. AR carried out laboratory experiments and supported data analysis. VG and SV collected the biological materials from animals. MG supported data analysis and drafted manuscript. LS participated in study coordination and supported the drafted manuscript. AM conceived and designed the study and participated in data analysis and drafted manuscript. All authors read and approved the final manuscript.

\section{Acknowledgements}

We thank Jose Luis Llergo for his excellent collaboration in parasite culture preparation.

\section{Competing interests}

The authors declare that they have no competing interests.

Received: 11 November 2015 Accepted: 1 March 2016

Published online: 08 March 2016

\section{References}

1. WHO. Methods for surveillance of antimalarial drug efficacy. Geneva: World Health Organization; 2009.

2. Daniels R, Volkman SK, Milner DA, Mahesh N, Neafsey DE, Park DJ, et al. A general SNP-based molecular barcode for Plasmodium falciparum identification and tracking. Malar J. 2008;7:223.

3. Li P, Zhao Z, Wang Y, Xing H, Parker DM, Yang Z, et al. Nested PCR detection of malaria directly using blood filter paper samples from epidemiological surveys. Malar J. 2014;13:175.

4. Menge DM, Ernst KC, Vulule JM, Zimmerman PA, Guo H, John CC. Microscopy underestimates the frequency of Plasmodium falciparum infection in symptomatic individuals in a low transmission highland area. Am J Trop Med Hyg. 2008;79:173-7.
5. Jimenez-Diaz MB, Mulet T, Viera S, Gomez V, Garuti H, Ibanez J, et al. Improved murine model of malaria using Plasmodium falciparum competent strains and non-myelodepleted NOD-scid IL2Rgammanull mice engrafted with human erythrocytes. Antimicrob Agents Chemother. 2009;53:4533-6.

6. Bahamontes-Rosa N, Bucher K, Held J, Robin A, Hoffmann WH, Flitsch SL, et al. In vivo anti-malarial effect of the beta-amino alcohol 1t on Plasmodium berghei. Parasitol Res. 2009;104:1459-64.

7. Ferrer P, Tripathi AK, Clark MA, Hand CC, Rienhoff HY Jr, Sullivan DJ Jr. Antimalarial iron chelator, FBS0701, shows asexual and gametocyte Plasmodium falciparum activity and single oral dose cure in a murine malaria model. PLoS ONE. 2012;7:e37171.

8. Kamau E, Tolbert LS, Kortepeter L, Pratt M, Nyakoe N, Muringo L, et al. Development of a highly sensitive genus-specific quantitative reverse transcriptase real-time PCR assay for detection and quantitation of Plasmodium by amplifying RNA and DNA of the $18 \mathrm{~S}$ rRNA genes. J Clin Microbiol. 2011;49:2946-53.

9. Hofmann N, Mwingira F, Shekalaghe S, Robinson LJ, Mueller I, Felger I. Ultra-sensitive detection of Plasmodium falciparum by amplification of multi-copy subtelomeric targets. PLoS Med. 2015;12:e1001788.

10. Murphy SC, Prentice JL, Williamson K, Wallis CK, Fang FC, Fried M, et al. Real-time quantitative reverse transcription PCR for monitoring of bloodstage Plasmodium falciparum infections in malaria human challenge trials. Am J Trop Med Hyg. 2012;86:383-94.

11. Jimenez-Diaz MB, Mulet T, Gomez V, Viera S, Alvarez A, Garuti H, et al. Quantitative measurement of Plasmodium-infected erythrocytes in murine models of malaria by flow cytometry using bidimensional assessment of SYTO-16 fluorescence. Cytometry A. 2009;75:225-35.

12. Sturm N, Hu Y, Zimmermann H, Fritz-Wolf K, Wittlin S, Rahlfs S, et al. Compounds structurally related to ellagic acid show improved antiplasmodial activity. Antimicrob Agents Chemother. 2009;53:622-30.

13. Witney AA, Doolan DL, Anthony RM, Weiss WR, Hoffman SL, Carucci DJ. Determining liver stage parasite burden by real time quantitative PCR as a method for evaluating pre-erythrocytic malaria vaccine efficacy. Mol Biochem Parasitol. 2001;118:233-45.

14. Shimizu S, Osada Y, Kanazawa T, Tanaka Y, Arai M. Suppressive effect of azithromycin on Plasmodium berghei mosquito stage development and apicoplast replication. Malar J. 2010;9:73.

15. Rae C, McQuillan JA, Parekh SB, Bubb WA, Weiser S, Balcar VJ, et al. Brain gene expression, metabolism, and bioenergetics: interrelationships in murine models of cerebral and noncerebral malaria. FASEB J. 2004;18:499-510

16. Su XZ, Carucci DJ, Wellems TE. Plasmodium falciparum: parasite typing by using a multicopy microsatellite marker, PfRRM. Exp Parasitol. 1998;89:262-5.

17. Korsinczky M, Chen N, Kotecka B, Saul A, Rieckmann K, Cheng Q. Mutations in Plasmodium falciparum cytochrome $b$ that are associated with atovaquone resistance are located at a putative drug-binding site. Antimicrob Agents Chemother. 2000;44:2100-8.

18. Angulo-Barturen I, Jimenez-Diaz MB, Mulet T, Rullas J, Herreros E, Ferrer $\mathrm{S}$, et al. A murine model of falciparum-malaria by in vivo selection of competent strains in non-myelodepleted mice engrafted with human erythrocytes. PLoS ONE. 2008;3:e2252.

19. Mawili-Mboumba DP, Nikiema R, Bouyou-Akotet MK, Bahamontes-Rosa $\mathrm{N}$, Traore A, Kombila M. Sub-microscopic gametocyte carriage in febrile children living in different areas of Gabon. Malar J. 2013;12:375.

20. Inoue J, Lopes D, Dov R, Machado M, Hristov AD, Lima GF, et al. Analysis of polymorphisms in Plasmodium falciparum genes related to drug resistance: a survey over four decades under different treatment policies in Brazil. Malar J. 2014;13:372.

21. Pritsch M, Wieser A, Soederstroem V, Poluda D, Eshetu T, Hoelscher $M$, et al. Stability of gametocyte-specific Pfs25-mRNA in dried blood spots on filter paper subjected to different storage conditions. Malar J. 2012;11:138.

22. Alemayehu S, Feghali KC, Cowden J, Komisar J, Ockenhouse CF, Kamau E. Comparative evaluation of published real-time PCR assays for the detection of malaria following MIQE guidelines. Malar J. 2013;12:277.

23. Strom GE, Tellevik MG, Hanevik K, Langeland N, Blomberg B. Comparison of four methods for extracting DNA from dried blood on filter paper for PCR targeting the mitochondrial Plasmodium genome. Trans R Soc Trop Med Hyg. 2014;108:488-94. 
24. Polley SD, Bell D, Oliver J, Tully F, Perkins MD, Chiodini PL, et al. The design and evaluation of a shaped filter collection device to sample and store defined volume dried blood spots from finger pricks. Malar J. 2015;14:45.
25. Murphy SC, Daza G, Chang M, Coombs R. Laser cutting eliminates nucleic acid cross-contamination in dried-blood-spot processing. J Clin Microbiol. 2012;50:4128-30.

Submit your next manuscript to BioMed Central and we will help you at every step:

- We accept pre-submission inquiries

- Our selector tool helps you to find the most relevant journal

- We provide round the clock customer support

- Convenient online submission

- Thorough peer review

- Inclusion in PubMed and all major indexing services

- Maximum visibility for your research

Submit your manuscript at www.biomedcentral.com/submit 